\title{
Investigation Of The Factors Affecting The Success Of Vestibular Rehabilitation Therapy In Patients With Idiopathic Unilateral Vestibular Hypofunction And Idiopathic Bilateral Vestibular Hypofunction
}

\author{
Suha Ertugrul MD1, - Emre Soylemez MSc²
}

\author{
1 Department of Otorhinolaryngology, Karabuk University, Faculty of Medicine, Karabuk, Turkey. ORCID ID:0000-0001-9743-6924 \\ 2 Department of Audiology, Karabuk University Training and Research Hospital, Karabuk, Turkey. ORCID ID:0000-0002-7554-3048
}

\begin{abstract}
Objective: The aim of this study was to determine the factors affecting the success of vestibular rehabilitation therapy (VRT) in patients with idiopathic unilateral vestibular hypofunction (UVH) and idiopathic bilateral vestibular hypofunction (BVH).

Methods: 30 patients with idiopathic UVH and 30 patients with idiopathic BVH were included in this prospective study. Visual analog scale (VAS) was used to evaluate the severity of the patients' complaints of dizziness. The duration of the complaint of dizziness was recorded. All patients underwent the Dizziness Handicap Inventory (DHI) before and after VRT. The severity, duration, and localization (unilateral/bilateral) of the vestibular hypofunction were compared with the efficacy of VRT.
\end{abstract}

Results: A significant decrease in DHI scores was observed after VRT for both, UVH and BVH patients, as compared to their pre-VRT scores $(p<0.001)$. There was no significant difference in the efficacy of vestibular rehabilitation therapy in patients with UVH and BVH $(p=0.09)$. As the VAS scores increased and the duration of the complaint lengthened, the efficacy of VRT decreased significantly $(p<0.001 / r=5.6, p=0.016 / r=3.1$, respectively).

Conclusion: VRT is an effective treatment for the relief of symptoms in both UVH and BVH patients. Unilateral or bilateral vestibular hypofunction does not affect the efficacy of VRT, whereas prolonged or severe symptoms of dizziness affect the effectiveness of VRT negatively. In idiopathic vestibular hypofunction patients with long-standing and/or severe dizziness complaints, VRT should be initiated immediately and continued longer.

Keywords: Vestibular diseases, dizziness, caloric tests, rehabilitation.

\section{Introduction}

Although dizziness is one of the most common health problems, not much is known about its etiology. ${ }^{[1,2]}$ The reason is that the causes could vary; neurological, cardiovascular, psychological, or vestibular problems may give rise to this particular complaint. ${ }^{[3,4]}$ Dizziness can cause anxiety and

depression and significantly affect the quality of life of the patients. Diseases such as Meniere's disease, vestibular neuritis, and meningitis may damage the vestibular organ and cause dizziness. Vestibular damage decreases the vestibulo-ocular reflex and vestibular hypofunction develops.
Correspondence: Suha Ertugrul

Sirinevler mahallesi, Alpaslan caddesi, no: 1, Merkez, Karabuk, Turkey

e-mail: drsuhaertugrul@hotmail.com

Received: 25.05.2019; Accepted: 17.07.2019
Online available at: www.entupdates.org

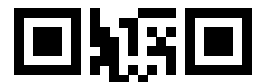

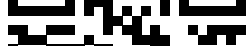
rentar

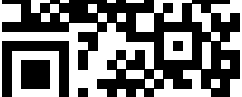


Vestibular hypofunction can develop rapidly progressive or slowly progressive and may affect unilateral ear or bilateral ears. Patients with vestibular hypofunction experience an imbalance that occurs with sudden movements of the head. These patients may also experience oscillopsia, a feeling of dizziness, and the feeling of being pushed. ${ }^{[5]}$ The vestibular hypofunction is diagnosed with tests that evaluate vestibulo-ocular reflexes such as video head impulse test, rotary chair, and caloric test. However, the cause of vestibular hypofunction may not be fully diagnosed in some cases such as patients with chronic dizziness. Lucieer et al ${ }^{[6]}$ found the definitive etiology in $47 \%$ of the 154 patients diagnosed with vestibular hypofunction and the possible etiology in $22 \%$. However, $31 \%$ of the patients could not find the etiology and considered as idiopathic vestibular hypofunction.

As pharmacotherapy provides a limited improvement in patients suffering from dizziness, vestibular rehabilitation therapy (VRT) is considered more suitable for them. ${ }^{[7-9]}$ The aim of VRT is to ensure static and dynamic stability by accelerating the neuroplasticity of the central nervous system and also increase the vestibulo-ocular interaction when contradictory sense inputs occur. Thus, the main purpose of the treatment is to minimize the patient's complaints and enhance the quality of life. Although VRT is an effective treatment modality in patients with dizziness, the factors affecting the efficacy of VRT are not known exactly. Also, to the best of our knowledge, there is no previous study that proves that the VRT program is more effective for either of the groups: patients with idiopathic unilateral vestibular hypofunction (UVH) or idiopathic bilateral vestibular hypofunction (BVH).

The aim of this study is to analyze the efficiency of VRT in adult patients with idiopathic UVH or idiopathic $\mathrm{BVH}$ and to detect which group benefits more from the VRT program. Another aim of this study is to determine the factors affecting the success of VRT in patients with idiopathic vestibular hypofunction.

\section{Methods}

\section{Patient selection}

In this prospective study, patients aged 18 years and over who were admitted to Otorhinolaryngology outpatient clinic with the complaint of dizziness were evaluated. Detailed anamnesis of all patients was obtained. Patients under 18 were excluded from the study. The patients were asked about the duration of dizziness and were recorded. Patients with dizziness complaints of less than three months were excluded from the study. Visual analog scale (VAS) was used to evaluate the severity of the patients' complaints of dizziness. The patients were asked to give a score between zero (no severity) and 10 (unbearable) for the severity of the symptoms of dizziness and the results were recorded. Patients with systemic disease and using the drug were also excluded from the study. All patients underwent an otorhinolaryngological examination. Patients with active infection focus, patients with chronic otitis media, patients with temporal or cranial fractures and patients with otologic surgery were excluded from the study. Patients with orthopedic disability were excluded from the study because they could affect VRT exercises. After the otorhinolaryngological examination, pure tone audiometry test and tympanometry test was applied to all of the patients. Patients with normal pure tone average, patients with tympanogram test type A and patients with normal acoustic reflex were included in the study. Patients who did not meet these criteria in the audiological tests were excluded from the study.

As a balance test, the Romberg test, oculomotor test, and caloric test were applied to all patients. In patients with suspected central pathology, central pathologies were excluded by cranial magnetic resonance and/or computed tomography. Patients with central pathology were excluded from the study. A slow phase rate of more than $25 \%$ nystagmus between two ears was considered as the criterion for the diagnosis of UVH in the caloric test. The following all three requirements were accepted as diagnostic criteria for BVH;

1- In the caloric test, the sum of nystagmus degrees after application of hot and cold air in both ears is 12 degrees/second and below. ${ }^{[10]}$

2- Seeing the objects as jump, jiggle or vibrate during the patient's head movements.

3- Positive Romberg test.

Vestibular hypofunction patients with known etiology were not included in the study. The study was continued with those patients who were diagnosed with idiopathic vestibular hypofunction, post these tests. All patients were applied to the Dizziness Handicap Inventory (DHI) (Table 1) and the VRT program was initiated thereafter. No addi- 
Table 1. Dizziness Handicap Inventory

Questions

Always

Sometimes

No

P1 Does looking up increase your problem?

E2 Because of your problem, do you feel frustrated?

F3 Because of your problem, do you restrict your travel for business or pleasure?

P4 Does walking down the aisle of a supermarket increase your problem?

F5 Because of your problem, do you have difficulty getting into or out of bed?

F6 Does your problem significantly restrict your participation in social activities, such as going out to dinner, going to movies, dancing or to parties?

F7 Because of your problem, do you have difficulty reading?

F8 Does performing more ambitious activities like sports, dancing, and household chores, such as sweeping or putting dishes away; increase your problem?

9 Because of your problem, are you afraid to leave your home without having someone accompany you?

E10 Because of your problem, have you been embarrassed in front of others?

P11 Do quick movements of your head increase your problem?

F12 Because of your problem, do you avoid heights?

P13 Does turning over in bed increase your problem?

F14 Because of your problem, is it difficult for you to do strenuous housework or yard work?

E15 Because of your problem, are you afraid people may think that you are intoxicated?

F16 Because of your problem, is it difficult for you to go for a walk by yourself?

P17 Does walking down a sidewalk increase your problem?

E18 Because of your problem, is it difficult for you to concentrate?

F19 Because of your problem, is it difficult for you to walk around your house in the dark?

E20 Because of your problem, are you afraid to stay home alone?

E21 Because of your problem, do you feel handicapped?

E22 Has your problem placed stress on your relationship with members of your family or friends?

E23 Because of your problem, are you depressed?

F24 Does your problem interfere with your job or household responsibilities?

P25 Does bending over increase your problem? 
tional medication was given to any patient, in addition to VRT. Patients were called for controls at second, fourth, and sixth weeks. The patients who had acute vertigo attack during the VRT process and those who did not complete the VRT program were excluded from the study. At the end of the sixth week, DHI had performed again for 30 $\mathrm{UVH}$ and $30 \mathrm{BVH}$ patients; these patients had completed the 6-week VRT program without experiencing a vertigo attack.

UVH and BVH patient groups were compared in terms of age, gender, duration of dizziness complaint and VAS scores. The efficiency of VRT was determined by comparing the pre-VRT and post-VRT DHI results. The relationship between age and gender and the efficacy of VRT was evaluated. The relationship between the severity, duration, and localization (unilateral/bilateral) of vestibular hypofunction and the effectiveness of the VRT program were evaluated.

\section{Assessment of hearing}

Clinical audiometry device (AC 40, DK-500; Interacoustics, Middelfart, Denmark) was used to evaluate the hearing. Pure-tone air conduction hearing threshold values of patients were measured at frequencies of $125,250,500$, $1000,2000,4000,6000$, and $8000 \mathrm{~Hz}$. Pure-tone bone conduction hearing threshold values of patients were measured at frequencies of 500,1000, 2000, 4000. The pure tone average was calculated by taking the average of hearing thresholds at frequencies of 500, 1000, 2000, and $4000 \mathrm{~Hz}$. The pure tone average of $20 \mathrm{~dB}$ and below was considered as a normal hearing. Speech recognition threshold and speech discrimination score tests were performed. Tympanometry measurement was performed with the impedance audiometer (AZ 26; Interacoustics, Middelfart, Denmark) in $226 \mathrm{~Hz}$ octave band by using $86 \mathrm{~dB}$ SPL probe tone stimulation. Type A tympanogram was accepted as normal (peaking at a pressure range of -100 to $50 \mathrm{dPa}$ ). Ipsilateral and contralateral acoustic reflex measurements were performed at 500, 1000, 2000 and $4000 \mathrm{~Hz}$.

\section{Caloric Test}

Micromedical brand (Spectrum 5, Chatham, USA) videonystagmography device was used for performing the caloric test. The test was performed in the supine position by putting the head of the patient at a 30 -degree flexion. The caloric test was done by applying hot $\left(47^{\circ} \mathrm{C}\right)$ and cold $\left(27^{\circ} \mathrm{C}\right)$ air to each ear for 1 minute. During caloric stim- ulation, in order to prevent the fixing of the patient's eyes, the lids of the glasses of the videonystagmography device were closed and the patient was asked to count to 50 . The best and cleanest nystagmus degrees were automatically analyzed and chosen by the computer. When artifacts were chosen as nystagmus by the computer, they were corrected manually.

\section{Romberg Test}

The Romberg test was performed on a hard floor for all patients. Patients were asked to stand with their feet together and their hands by their sides. Also, they were asked to close their eyes and the stopwatch was started. Romberg was considered negative if the patient was able to maintain his/her balance with the eyes closed for 30 seconds. Patients who could not maintain their balance for $30 \mathrm{sec}-$ onds and who opened their eyes were considered Romberg positive.

\section{Vestibular Rehabilitation Therapy}

Patients were administered the VRT program that consisted of 21 exercises such as vestibular adaptation, balance and coordination, vestibular habituation, and oculomotor exercises. After the patients had been diagnosed with idiopathic vestibular hypofunction, they were recommended the simplest VRT exercises three times a day with 10 sets each for 15 days. After their second and fourth week controls, the patients were given rehabilitation exercises that were more difficult than the previous exercise set. At the end of six weeks, the patients were expected to complete the rehabilitation program. The VRT exercises provided for the patients are given in Table 2.

\section{Dizziness Handicap Inventory (DHI)}

This is an inventory that consists of 25 questions. ${ }^{[11]}$ Nine of these questions are about the emotional condition of the patients, nine about the functional condition and seven are about their physical condition. For each question, a "No" gets zero points, a "Sometimes" gets two points, and a "Yes" is four points. The total score is calculated over 100 points. 0-16 points are evaluated as "no handicap", 18-34 points as "mild handicap", 36-52 points as "moderate handicap", and 54-100 points are evaluated as "severe handicap".

\section{Statistical Analysis}

We used SPSS version 21.0 statistical software (SPSS Inc.; 


\begin{tabular}{|c|c|c|}
\hline Time to start exercise & Started exercises & \\
\hline At the beginning & $\begin{array}{l}\text { Sitting and standing } \\
\text { While sitting on the chair, eyes open, head } \\
\text { right-left, up-down } \\
\text { While sitting on the chair, eyes closed, head } \\
\text { right-left, up-down } \\
\text { While sitting on the chair, head fixed, finger } \\
\text { follow, right-left, up-down }\end{array}$ & $\begin{array}{l}\text { While sitting on the chair, the finger is fixed and the eyes are sta- } \\
\text { ble on the finger, turning the head right-left, up-down } \\
\text { Bend down and up while sitting on a chair }\end{array}$ \\
\hline $\begin{array}{l}\text { First control } \\
\text { (Second week) }\end{array}$ & $\begin{array}{l}\text { While standing, eyes closed, head right-left, } \\
\text { up-down } \\
\text { While standing, eyes open, head right-left, } \\
\text { up-down } \\
\text { Eyes closed on a straight line assisted walking } \\
\text { Eyes open head right-left on a straight line } \\
\text { assisted walking (eyes not stable) }\end{array}$ & $\begin{array}{l}\text { Step counting with eyes closed } \\
\text { Eyes closed, head right-left while walking on a straight line } \\
\text { While standing, head fixed, finger follow, right-left, up-down } \\
\text { While standing, the finger is fixed and the eyes are stable on the } \\
\text { finger, turning the head right-left, up-down }\end{array}$ \\
\hline $\begin{array}{l}\text { Second control } \\
\text { (Fourth week) }\end{array}$ & $\begin{array}{l}\text { Quickly lying first right then left side while } \\
\text { sitting } \\
\text { Throwing a ball to wall and catching back } \\
\text { while standing } \\
\text { Go up and down stairs with eyes closed } \\
\text { In the tandem position, eyes open, head } \\
\text { right-left, up-down }\end{array}$ & $\begin{array}{l}\text { In the tandem position, head fixed, finger follow, right-left, up- } \\
\text { down } \\
\text { In the tandem position, the finger is fixed and the eyes are stable } \\
\text { on the finger, turning the head right-left, up-down } \\
\text { While walking, the eyes are fixed to a point while turning the } \\
\text { head to the left and right }\end{array}$ \\
\hline
\end{tabular}

Chicago, IL, USA) to perform our analyses. For descriptive statistics, percent, mean, standard deviation (SD), median, minimum and maximum values were presented. T-test was used to evaluate age and sex distribution among the UVH patients and BVH patients. The Mann Whitney-U test was used to evaluate the duration of dizziness and VAS scores between the UVH patients and BVH patients. In order to evaluate the DHI difference between pre-VRT and postVRT, the importance of the distinction between the paired t-test was used.

The relationship between age and the efficacy of the VRT was examined by Pearson correlation test and the relationship between sex and the efficacy of the VRT was examined with $\mathrm{t}$-test. The t-test was used to compare the efficacy of the VRT program between patients with UVH and BVH. Spearman correlation test was used to evaluate the relationship between duration of dizziness, VAS score, and benefits of the VRT.

\section{Ethical Situation}

Permission for this study was granted from the local ethics committee of the institution (2018-3/10). All patients were informed about the procedures and written informed consent forms were received. The study has been performed in accordance with the principles of the Helsinki Declaration.

\section{RESULTS}

Forty-four (73.3\%) of the 60 patients involved in the study were female and $16(16.7 \%)$ of them were male, with the average age being $46.58 \pm 14.44$ years (range, $19-85$ years). Pure tone audiometry test and oculomotor tests were normal in all patients included in the study. Of the $30 \mathrm{UVH}$ patients, 14 had oscillopsia. All patients with BVH had oscillopsia. The difference between the responses of both ears in the caloric test in patients with UVH was an average of $41.20 \pm 14.63 \%$ (range, $25 \%-67 \%$ ). There was no significant difference between the patients with UVH and those with BVH in terms of age, gender, duration of dizziness, and VAS scores ( $\mathrm{p}=0.075, \mathrm{p}=0.24, \mathrm{p}=0.406, \mathrm{p}=0.551$, respectively). Age, gender and duration of dizziness distribution of the groups are given in Table 3 . According to DHI results, 24 UVH patients (80\%) and $24 \mathrm{BVH}$ patients (80\%) had moderate to severe disability before VRT was employed. However, after the VRT program, these numbers reduced to six patients (20\%) and 10 patients (33.3\%), respectively (Table 4). A significant decrease in DHI scores was observed after VRT for both, UVH and BVH patients, as compared to their pre-VRT scores $(\mathrm{p}<0.001)$. The 


\begin{tabular}{|c|c|c|c|}
\hline & UVH group & BVH group & P-value \\
\hline Age (years) & & & $0.075^{\mathrm{a}}$ \\
\hline Mean \pm SD & $49.9 \pm 11.88$ & $43.26 \pm 16.14$ & \\
\hline Min-Max & $19-78$ & $20-85$ & \\
\hline $\operatorname{Sex}(n)$ & & & $0.24^{b}$ \\
\hline Male & 6 & 10 & \\
\hline Female & 24 & 20 & \\
\hline Benefit of VRT & $32.06 \pm 12.77$ & $26(6-52)$ & $0.090^{c}$ \\
\hline Duration of dizziness (month) & $10(3-72)$ & $12(3-84)$ & $0.406^{b}$ \\
\hline \multicolumn{4}{|l|}{ Median (min-max) } \\
\hline VAS score & $6.3 \pm 1.7$ & $6.8 \pm 1.9$ & $0.551^{\mathrm{a}}$ \\
\hline
\end{tabular}

a T-test

${ }^{\mathrm{b}}$ chi-square test

'Mann Whitney-U test

Table 4. Disability severity distribution of patients with unilateral vestibular hypofunction (UVH) and bilateral vestibular hypofunction (BVH) before and after vestibular rehabilitation therapy (VRT).

\begin{tabular}{lcccc}
\hline Severity of disability & \multicolumn{2}{c}{ Before VRT } & \multicolumn{2}{c}{ After VRT } \\
\hline UVH group & $\mathbf{n}$ & $\%$ & $\mathbf{n}$ & $\%$ \\
\cline { 2 - 5 } Normal & 0 & 0.0 & 15 & 50 \\
Mild & 6 & 20 & 9 & 30 \\
Moderate & 11 & 36.6 & 5 & 16.6 \\
Severe & 13 & 43.3 & 1 & 3.3 \\
BVH group & & & & \\
Normal & 0 & 0.0 & 13 & 43.3 \\
Mild & 6 & 20 & 9 & 30 \\
Modarate & 10 & 33.3 & 3 & 10 \\
Severe & 14 & 46.6 & 5 & 16.6 \\
\hline
\end{tabular}

scores of functional, emotional and physical components of patients with UVH and BVH were similar before VRT administration. Significant improvement was observed in all components after the VRT program was applied $(\mathrm{p}<0.001)$. However, the functional disability scores were higher than emotional and physical disability scores for both patient groups (Table 5).
There was no significant difference between the UVH patients and BVH patients in terms of the benefits of the VRT program $(\mathrm{p}=0.09)$. There was no significant difference in the efficacy of VRT with age and gender $(\mathrm{p}=0.639$, $\mathrm{p}=0.083$, respectively). There was a negative correlation between the duration of dizziness $(\mathrm{p}=0.016, \mathrm{r}=3.1)$ and VRT efficacy and between the VAS and VRT efficiency $(\mathrm{p}<0.001, \mathrm{r}=5.6)$.

\section{Discussion}

In peripheral vestibular hypofunction, there is a decrease in the amount of neural stimulus reaching the vestibular nucleus, resulting in an asymmetry. ${ }^{[9]}$ As a result, dizziness at head movements, oscillopsia, and postural instability develop. ${ }^{[12,13]}$ It is known that the VRT program is used effectively in the treatment of dizziness and vertigo. ${ }^{[14-17]} \mathrm{Kulcu}$ et al ${ }^{[14]}$, in their study of 38 patients with benign paroxysmal positional vertigo, found that VRT program was more effective than drug (betahistine) treatment and suggested that VRT could be preferred in chronic and permanent vertigo patients. Ribeiro et al ${ }^{[15]}$ found that VRT in addition to canalith repositioning maneuver in benign paroxysmal positional vertigo patients provided better dynamic balance results. In another study, Sahin et al ${ }^{[16]}$ performed a VRT program for 17 patients who were diagnosed with BVH for six weeks and reported that the VRT program was an effective treatment in patients with BVH and in- 


\begin{tabular}{|c|c|c|c|c|}
\hline \multirow[t]{2}{*}{ Components of DHI } & \multicolumn{2}{|c|}{ UVH group } & \multicolumn{2}{|c|}{ BVH group } \\
\hline & Before VRT & After VRT & Before VRT & After VRT \\
\hline Functional score & $22.8 \pm 8.68$ & $8(0-32)$ & $22.87 \pm 9.59$ & $9(0-32)$ \\
\hline Emotional score & $12.87 \pm 6.74$ & $2(0-22)$ & $12.20 \pm 7.36$ & $2(0-22)$ \\
\hline Physical score & $16(0-24)$ & $6.47 \pm 4.68$ & $15.73 \pm 4.57$ & $8(0-20)$ \\
\hline Total Score & $51.2 \pm 14.48$ & $15(0-60)$ & $50.8 \pm 17.9$ & $21(0-66)$ \\
\hline P-value & \multicolumn{2}{|c|}{$<0.001^{a}$} & \multicolumn{2}{|c|}{$<0.001^{a}$} \\
\hline
\end{tabular}

Values represent mean \pm standart deviation or median (min-max), UVH: unilateral vestibular hypofunction, BVH: bilateral vestibular hypofunction a Paired t-test

creased the efficiency of treatment when the rehabilitation duration increased. Giray et al ${ }^{[17]}$ investigated the efficacy of the VRT program in patients with chronic UVH and found that the VRT program improved the symptom, postural stability, balances and disability of UVH patients significantly. Although it has been demonstrated in the studies that the patients with UVH and BVH benefit from the VRT program, it has not been previously investigated which group of patients benefit more than the VRT program to the best of our knowledge. Similar to the literature, we found that both UVH patients and BVH patients benefited significantly from the VRT program. However, there was no significant difference in the efficacy of VRT in UVH or BVH patients.

The severity of the complaint of dizziness may not always be consistent with the patient's caloric test responses. The degree of vestibular hypofunction that causes dizziness in patients is different. The ability to cope with dizziness is also different. This may be due to individuals' genetic characteristics, lifestyles or environmental factors. Bamiou et al ${ }^{[18]}$ investigated the severity of dizziness of patients with partial canal paresis and patients with total canal paresis and found no difference between the two groups. In this study, we determined the severity of dizziness complaints using VAS. There was no difference in VAS scores between patients with UVH and BVH. In our study, UVH or BVH did not affect the efficacy of VRT, whereas high VAS scores had a negative effect on the efficacy of the VRT program. Patients with severe dizziness complaints benefit significantly from VRT, so longer-term VRT may be applied to such patients than patients with mild complaints of dizziness.

Bayat et al ${ }^{[9]}$ applied a VRT program to elderly patients with chronic UVH for 8 weeks. They reported that the effect of the program was seen immediately and the physical and functional scores of the patients were close to each other and higher than the emotional status scores. ${ }^{[9]}$ In our study, we found that the physical and functional status of patients with $\mathrm{UVH}$ and patients with $\mathrm{BVH}$ were close to each other but more affected by the emotional situation. The emotional component questionnaire consisted of queries relating to patients being afraid of staying alone at home, feeling ashamed when they are with other people due to their problem with dizziness, and because of dizziness, are you afraid people may think that you are intoxicated. The chronic dizziness patients in our study reconciled to life with complaints of dizziness; none of the patients who completed the study gave any score to item 15 . Lin et al ${ }^{[19]}$ applied DHI in dizzy patients and they found that functional component the most difficult for unsteady patients. Giray et al ${ }^{[17]}$ classified patients with unilateral vestibular hypofunction in two groups and reported that the group to whom the VRT program was administered had a lower level of disability than the control group. Moreover, they found that patients with dizziness had more difficulty in the functional component score as compared to the emotional and physical components scores similar to our study. However, taking into account the fact that DHI has seven questions with which to evaluate the physical score of the patients but nine questions to evaluate their functional and emotional scores. We are of the opinion that the reason for this difference lies in the two extra questions that were asked to gauge the emotional and functional scores.

In our study, we found that age and gender did not affect the efficacy of the VRT program, similar to the literature. ${ }^{[20]}$ Although the rate of recovery and neural plasticity are 
faster in the young than in the elderly, it is interesting that the efficacy of VRT does not change with age. This may be due to the more sedentary life of the elderly patients and the lower expectations of the elderly. Even though the elderly have benefited less than the VRT program according to the young people, they may have benefited enough to continue their daily lives.

The VRT program is known to be effective in individuals with both acute and chronic dizziness. ${ }^{[9,21]}$ However, there are conflicting results in terms of the relationship between the duration of dizziness and the benefit of the VRT program in the literature. ${ }^{[20,22,23]}$ Herdman et al ${ }^{[20]}$ did not find a relationship between the duration of dizziness and the benefit of the VRT program in patients with UVH. However, Bamiou et al ${ }^{[23]}$ found that individuals who started to VRT program in the first 6 months had more benefits than those who started later. We compared the efficacy of the VRT program with the duration of dizziness in patients with UVH and patients with BVH who had been suffering from dizziness for more than three months, and we found that the effectiveness of VRT decreased significantly as the duration of the dizziness complaint increased. The effec-

\section{References}

1. Murdin L, Schilder AG. Epidemiology of balance symptoms and disorders in the community: a systematic review. Otol Neurotol 2015;36:387-92.

2. Yardley L, Owen N, Nazareth I, Luxon L. Prevalence and presentation of dizziness in a general practice community sample of working age people. Br J Gen Pract 1998;48:1131-5.

3. Chan Y. Differential diagnosis of dizziness. Curr Opin Otolaryngol Head Neck Surg 2009;17:200-3.

4. Karatas M. Central vertigo and dizziness: epidemiology, differential diagnosis, and common causes. Neurologist 2008;14:355-64.

5. Han BI, Song HS, Kim JS. Vestibular rehabilitation therapy: review of indications, mechanisms, and key exercises. J Clin Neurol 2011;7:184-96.

6. Lucieer F, Vonk P, Guinand N, Stokroos R, Kingma H, van de Berg R. Bilateral vestibular hypofunction: insights in etiologies, clinical subtypes, and diagnostics. Front Neurol 2016;7:26.

7. Martins E, Silva DC, Bastos VH, et al. Effects of vestibular rehabilitation in the elderly: a systematic review. Aging Clin Exp Res 2016;28:599-606.

8. Ricci NA, Aratani MC, Doná F, Macedo C, Caovilla HH, Ganança FF. A systematic review about the effects of the vestibular rehabilitation in middle-age and older adults. Rev Bras Fisioter 2010;14:361-71.

9. Bayat A, Saki N. Effects of Vestibular Rehabilitation Interventions in the Elderly with Chronic Unilateral Vestibular Hypofunction. Iran J Otorhinolaryngol 2017;29:183-8.

10. Katz J, Chasin M, English K, Hood L, Tillery K. Handbook of clinical audiology, seventh edition, Philadelphia: Wolters Kluwer; 2015. pp. 414. tiveness of VRT may be less because patients with a long history of dizziness may have more secondary problems such as anxiety and depression.

To apply objective test methods like posturography before and after the VRT is likely to provide beneficial information in additional studies.

\section{Conclusion}

It has been concluded that the VRT has an effective role in eliminating the symptoms of both $\mathrm{UVH}$ and $\mathrm{BVH}$ patients and is seen to decrease the severity of the disability. However, there is no significant difference in the efficacy of VRT in patients with UVH and BVH. In patients with chronic imbalance, the efficacy of VRT was decreased when the severity and duration of the imbalance complaint increased. Therefore, VRT should be initiated immediately in patients with vestibular hypofunction and VRT should be kept longer in patients with severe and long-term complaints of imbalance.

Conflicts of interest: The authors report no conflicts of interest.
11. Jacobson GP, Newman CW. The development of the Dizziness Handicap Inventory. Arc Otolaryngol Head Neck Surg 1990;116:424-7.

12. Schubert MC, Minor LB. Vestibulo-ocular physiology underlying vestibular hypofunction. Phys Ther 2004;84:373-85.

13. Hillier $\mathrm{S}, \mathrm{McD}$ onnell $\mathrm{M}$. Is vestibular rehabilitation effective in improving dizziness and function after unilateral peripheral vestibular hypofunction? An abridged version of a Cochrane review. Eur J Phys Rehabil Med 2016;52:541-56.

14. Kulcu DG,Yanik B, Boynukalin S, Kurtais Y. Efficacy of a home-based exercise program on benign paroxysmal positional vertigo compared with betahistine. J Otolaryngol Head Neck Surg 2008;37:373-9.

15. Ribeiro KM, Freitas RV, Ferreia LM, Deshpande N, Guerra RO. Effects of balance vestibular rehabilitation on therapy in elderly with benign paroxysmal positional vertigo: a randomized contrrolled trial. Disabil Rehabil 2017;39:1198-206.

16. Şahin E, Dinç ME, Yayla Özker B, Çöpürgensli C, Konaklığlu M, Özçelik T. The value of vestibular rehabilitation in patients with bilateral vestibular dysfunction. J Int Adv Otol 2017;13:385-9.

17. Giray M, Kirazli Y, Karapolat H, Celebisoy N, Bilgen C, Kirazli T. Shortterm effects of vestibular rehabilitation in patients with chronic unilateral vestibular dysfunction: a randomized controlled study. Arch of Phys Med Rehabil 2009;90:1325-31. 
18. Bamiou DE, Davies RA, McKee M, Luxon LM. The effect of severity of unilateral vestibular dysfunction on symptoms, disabilities and handicap in vertiginous patients. Clin Otolaryngol Allied Sci 1999;24:31-8.

19. Lin SI, Tsai TT, Lee IH, Wu YN. Perception of unsteadiness in patients with dizziness: association with handicap and imbalance. J Biomed Sci 2002;9:428-35.

20. Herdman SJ, Hall CD, Delaune W. Variables associated with outcome in patients with unilateral vestibular hypofunction. Neurorehabil Neural Repair 2012;26:151-62.

21. Strupp M, Arbusow V, Maag KP, Gall C, Brandt T. Vestibular exercises improve central vestibulospinal compensation after vestibular neuritis. Neurology 1998;51:838-44.

22. Telian S, Shepard N, Smith-Wheelock M, Kemink J. Habituation therapy for chronic vestibular dysfunction: preliminary results. Otolaryngol Head Neck Surg 1990;103:89-95

23. Bamiou DE, Davies RA, McKee M, Luxon LM. Symptoms, disability and handicap in unilateral peripheral vestibular disorders. Effects of early presentation and initiation of balance exercises. Scand Audiol 2000;29:238-44.

This is an open access article distributed under the terms of the Creative Commons Attribution-NonCommercial-NoDerivs 3.0 Unported (CC BY- NC-ND3.0) Licence (http://creativecommons.org/licenses/by-nc-nd/3.0/) which permits unrestricted noncommercial use, distribution, and reproduc- tion in any medium, provided the original work is properly cited.

Please cite this article as: Ertugrul S, Soylemez E. Investigation Of The Factors Affecting The Success Of Vestibular Rehabilitation Therapy In Patients With Idiopathic Unilateral Vestibular Hypofunction And Idiopathic Bilateral Vestibular Hypofunction. ENT Updates 2019;9(2): 150-158 